\title{
Escaping the Plato's cave of latent tuberculosis testing: a path for developers of predictive tests for risk of tuberculosis
}

\author{
Morten Ruhwald ${ }^{1}$ and Roland Diel (1) ${ }^{2,3}$ \\ Affiliations: ${ }^{1}$ Center for Vaccine Research, Statens Serum Institut, Copenhagen, Denmark. ${ }^{2}$ Institute for \\ Epidemiology, University Medical Hospital Schleswig-Holstein, Kiel, Germany. ${ }^{3}$ Lung Clinic Grosshansdorf, \\ Airway Disease Center North (ARCN), German Center for Lung Research (DZL), Grosshansdorf, Germany.
}

Correspondence: Morten Ruhwald, Center for Vaccine Research, Statens Serum Institut, Artillerivej 5, 2300 Copenhagen, Denmark. E-mail: morudssi.dk

@ERSpublications

The End TB Strategy calls for renewed focus on latent TB, which requires development of more predictive tests. KIK et al. provide a framework for the clinical development of such tests, an ambition that calls for joint efforts by researchers and funders. http://ow.ly/O3tm30m95dy

Cite this article as: Ruhwald M, Diel R. Escaping the Plato's cave of latent tuberculosis testing: a path for developers of predictive tests for risk of tuberculosis. Eur Respir J 2018; 52: 1801616 [https://doi.org/ 10.1183/13993003.01616-2018].

\section{The End TB Strategy and current state of play}

Tuberculosis (TB) is one of the leading causes of morbidity and death, disproportionately impacting the low- and middle-income countries, where $94 \%$ of TB cases occur. Every year, TB causes more than a million deaths and the World Bank estimates the epidemic will rob the world's poorest countries of US\$1-3 trillion over the next decade, causing a loss of productivity of up to $4-7 \%$ of some countries' gross domestic product. Particularly troubling for the prospects of global prosperity, $75 \%$ of TB cases arise during people's most productive years, between the ages of 15 and 54, stifling human development on a large scale.

The End TB Strategy, introduced by the World Health Organization (WHO) in 2015, aims to reduce TB-related deaths by $95 \%$ and new Mycobacterium tuberculosis infections by $90 \%$ by 2035 . These ambitious targets will be achieved, in part, by addressing latent $M$. tuberculosis infection (LTBI), the asymptomatic and noncontagious form of the disease estimated to affect one in four of the global population [1-3]. Mathematical modelling studies focusing on the WHO Southeast Asia region suggest that TB incidence could be reduced dramatically by 2050 by targeting LTBI testing and treatment [4]. However, a major challenge to the success of the strategy is that there is currently no test or biomarker able to correctly pick out the $5-15 \%$ of infected individuals who, at any point in their lifetime, will progress to TB disease from the pool of individuals who will not progress.

The current diagnostic landscape comprises tests that determine infection based on an immune response to mycobacterial antigens either in vivo, as in the tuberculin skin test (TST), or in vitro using the interferon- $\gamma$ release assays (IGRAs) [2]. Much like the prisoners in Plato's cave, only able to see the shadows of things on the wall and not the actual things themselves, these tests provide treating physicians with an indication of current or past infection and not a direct verification of the bacterium or risk of disease. The TST was 
developed by pioneers including Koch, Mantoux and von Piquet in the infancy of modern medicine. The test measures a delayed-type hypersensitivity skin response to tuberculin purified protein derivative and has well known challenges in terms of unspecific responses in bacille Calmette-Guérin-vaccinated people and people exposed to environmental mycobacteria. The IGRAs, developed in the late 1990s [5], measure in vitro reactivity of immune cells from the blood to antigens largely restricted to the $M$. tuberculosis complex organisms. IGRAs are thus more specific, and deliver faster and more objective results than the TST; however, they require more resources and are more expensive [2]. As evident from several meta-analyses, both IGRAs and the TST have only limited ability to predict the risk of progression to disease (positive predictive value (PPV)). In one study, the pooled PPV was $2.7 \%$ (95\% CI 2.3-3.2\%) for IGRAs and only $1.5 \%(95 \%$ CI $1.2-1.7 \%)$ for TST [1]. Another meta-analysis, mainly focusing on long-term follow-up in highly endemic settings, showed a pooled unadjusted incidence rate of $2.1 \%(95 \%$ CI 1.4-3.1\%) for IGRA [6]. In adolescent children, the pooled PPV for progression was only moderate with a PPV of $6.8 \%$ for the IGRAs compared to $2.4 \%$ for the TST [7]. Thus, even when applying the best current diagnostic options in high risk populations in low incidence settings, the numbers needed to treat to prevent a case of TB remain well above 30, and even higher in high-incidence settings [2, 8, 9]. With these numbers in mind, the WHO End TB Strategy's focus on prevention of progression would seem overly optimistic, if not naïve.

\section{A bold vision and an escape from Plato's cave?}

The WHO's strategy sets a bold vision for a future with improved predictive tools enabling more accurate and cost-effective test and treatment strategies. The vision is driven by the availability of shorter treatment regimens (e.g. using a 12-dose once-weekly rifapentine and isoniazid combination [10]), but in particular also by a recognition that the old binary paradigm of "LTBI" and "active TB disease" has fallen and new diagnostic opportunities seem within the technical reach of developers [11, 12].

Recent advances using cutting-edge animal models, immunology, microbiology and imaging tools support the existence of an infection spectrum from spontaneous clearance to quiescent infection and active TB disease $[11,13]$. The infected individuals' position in this spectrum is determined by the ability to control bacillary replication, which again is dynamic and influenced by factors such as nutritional status, HIV infection and compliance to anti-TB treatment $[11,13]$. The spectrum paradigm includes a central postulate that, prior to clinical presentation with active $\mathrm{TB}$ disease, there might be a prolonged asymptomatic phase of incipient TB (subclinical disease), during which pathology develops [11, 12].

Based on these assumptions, diagnostic tests for the identification of latent infection can conceptually be categorised as persistent infection tests (PITs) (a space largely covered by the current diagnostic portfolio for LTBI) and tests detecting incipient TB (ITTs) [12, 14]. A therapeutic intervention directed by ITTs (with or without the requirement of a two-step approach with an initial rule-out PIT) would be the most cost-effective and safe approach to programmatic management of LTBI,the "holy grail" for test developers.

These diagnostic concepts were discussed and developed at a series of Technical Expert Consultations, hosted by the Foundation for Innovative New Diagnostics (FIND), Stop TB Partnership's New Diagnostic Working Group (NDWG) and the WHO Global TB Program, as summarised in an outline for a Target Product Profile and Evaluation Framework for "a test predicting progression from tuberculosis infection to active disease" [8], a highly relevant initiative, aiming to inspire researchers and test developers to rethink the field of biomarkers for LTBI.

\section{A gameplan for action}

In this issue of the European Respiratory Journal, KIK et al. [15] expand on the evaluation framework by describing the requirements to support a Grading of Recommendations Assessment, Development and Evaluation (GRADE) evaluation process at WHO to inform policy guidance for new ITTs. The authors describe the study designs needed to document performance characteristics of such tests and the types of studies required to document the impact on patient and health system outcomes. The clinical development plans for ITTs require a radically different ambition from the current standard of care in the field, where many, often underpowered, studies claim diagnostic potential based on a significant p-value between groups of IGRA-positive cases and controls. In contrast, due to the elusive nature of incipient TB, ITT studies are very large and will require multicentre and likely multinational prospective efforts.

Nevertheless, there is room for optimism. The prognostic correlate of risk (COR), a predictive test enabling risk stratification based on selected inflammatory patterns in the mRNA transcriptome, provide a tangible case story for the likelihood of success with this strategy [16]. The COR materialised after joining cohorts and minds across several large international consortia funded by the European Union and the Bill and Melinda Gates Foundation, among others. Although the PPV of the COR still remain sub-par in the eyes of the ITT Target Product Profile $[8,17]$, its potential to guide preventive treatment is currently under 
evaluation at several sites in South Africa in the Correlate of Risk Targeted Intervention Study (www. clinicaltrials.gov identifier number NCT02735590). Results from this trial are expected by the end of 2019 (M. Hatherill, University of Cape Town, Cape Town, South Africa; personal communication) and will be a litmus test for the ITT pursuit and, at least in part, provide an interim evaluation of the likelihood of success of the End TB Strategy's focus on LTBI.

Until we have proven efficacy and WHO endorsement of new ITTs, we will have to make do with the current diagnostic portfolio and incremental improvements hereof [2]. Recent findings from large prospective cohorts in Norway [18] and South Africa [19] suggest superior risk stratification in algorithms incorporating the magnitude of the interferon (IFN)- $\gamma$ response in the IGRA [20] and serial IGRA testing. Other approaches seek to improve the TST and IGRA technologies, such as field-friendly specific skin tests including C-Tb based on the antigens used in IGRAs [21], and lateral flow or quantitative RT-PCR based tests using highly expressed markers like IFN- $\gamma$-inducible protein 10 as an alternative to IFN- $\gamma$ [22], potentially combined with novel antigens to improve sensitivity in high-risk groups such as young children and people living with HIV [23, 24].

\section{Perspectives}

The concrete guidance for clinical development plans laid out in the article by KIK et al. [15] underpins a requirement of radically new and more ambitious approaches to test development. An obvious place to start would be to integrate ITT development into TB drug and, in particular, TB vaccine trials, where two vaccine candidates recently have demonstrated protective efficacy and two other vaccines are ongoing efficacy assessment also with disease end-points $[25,26]$. The ability to integrate initiatives would support and improve immunogenicity assessment of the vaccines as well as being a very cost-effective approach to prospectively build biobanks for ITT test development.

The spectrum paradigm, new research tools becoming available in the near future and tangible evidence suggesting a future with ITT has brought renewed enthusiasm and inspired the incentives from WHO, NDWG and FIND. It is now up to the research community and funders to join forces and deliver.

Conflict of interest: M. Ruhwald is an employee of Statens Serum Institut (SSI), Copenhagen, Denmark, outside the submitted work. In addition, he has patent WO2017084671A1 pending and patent WO2008028489A2 issued, and he has further served as Industry Representative at the Technical Expert Consultations supporting the development of the TPP for predictive tests. In January 2017, SSI divested the production of RT-23 purified protein derivative (PPD) for the TST to AJ Vaccines, hereby eliminating a potential conflict of interest for the RT-23 PPD TST. R. Diel reports receiving fees and/or travel reimbursement from Cellestis, Oxford Immunotec and Pharmore, and travel reimbursement for serving on an advisory board from Generium, outside the submitted work.

Support statement: M. Ruhwald receives funding from the European Commission Horizon 2020 programme (grant number TBVAC2020 643381) and Research Council Norway (GLOBVAC 248042/H10). Funding information for this article has been deposited with the Crossref Funder Registry.

\section{References}

1 Diel R, Loddenkemper R, Nienhaus A. Predictive value of interferon- $\gamma$ release assays and tuberculin skin testing for progression from latent TB infection to disease state, a meta-analysis. Chest 2012; 142: 63-75.

2 Pai M, Denkinger CM, Kik SV, et al. Gamma interferon release assays for detection of Mycobacterium tuberculosis infection. Clin Microbiol Rev 2014; 27: 3-20.

3 Houben RM, Dodd PJ. The global burden of latent tuberculosis infection: a re-estimation using mathematical modelling. PLOS Med 2016; 13: e1002152.

4 Abu-Raddad LJ, Sabatelli L, Achterberg JT, et al. Epidemiological benefits of more-effective tuberculosis vaccines, drugs, and diagnostics. Proc Natl Acad Sci USA 2009; 106: 13980-13985.

5 Andersen P, Munk ME, Pollock JM, et al. Specific immune-based diagnosis of tuberculosis. Lancet 2000; 356 1099-1104.

6 Rangaka MX, Wilkinson KA, Glynn JR, et al. Predictive value of interferon- $\gamma$ release assays for incident active tuberculosis: a systematic review and meta-analysis. Lancet Infect Dis 2012; 12: 45-55.

7 Machingaidze S, Verver S, Mulenga $\mathrm{H}$, et al. Predictive value of recent QuantiFERON conversion for tuberculosis disease in adolescents. Am J Respir Crit Care Med 2012; 186: 1051-1056.

8 Boehme C, Churchyard G, Cobelens F, et al. Development of a Target Product Profile (TPP) and a framework for evaluation for a test for predicting progression from tuberculosis infection to active disease. www.finddx.org/ wp-content/uploads/2017/10/TPP-framework-for-evaluation-test-predicting-progression-from-TB-active-disease_ 2017.pdf. Date last updated: 2017. Date last accessed: August 21, 2018.

9 Matteelli A, Sulis G, Capone S, et al. Tuberculosis elimination and the challenge of latent tuberculosis. La Presse Médicale 2017; 46: e13-e21.

10 Sterling TR, Villarino ME, Borisov AS, et al. Three months of rifapentine and isoniazid for latent tuberculosis infection. N Engl J Med 2011; 365: 2155-2166.

11 Esmail H, Barry CE, Young DB, et al. The ongoing challenge of latent tuberculosis. Phil Trans R Soc B 2014; 369: 20130437. 
12 Drain PK, Bajema KL, Dowdy D, et al. Incipient and subclinical tuberculosis: a clinical review of early stages and progression of infection. Clin Microbiol Rev 2018; 31: e00021-18.

13 Cadena AM, Fortune SM, Flynn JL. Heterogeneity in tuberculosis. Nat Rev Immunol 2017; 17: 691-702.

14 Cobelens F, Kik S, Esmail H, et al. From latent to patent: rethinking prediction of tuberculosis. Lancet Respir Med 2017; 5: 243-244.

15 Kik SV, Shumacher S, Cirillo DM, et al. An evaluation framework for new tests that predict progression from tuberculosis infection to clinical disease. Eur Respir J 2018; 52: 1800946.

16 Zak DE, Penn-Nicholson A, Scriba TJ, et al. A blood RNA signature for tuberculosis disease risk: a prospective cohort study. Lancet 2016; 387: 2312-2322.

17 Petruccioli E, Scriba TJ, Petrone L, et al. Correlates of tuberculosis risk: predictive biomarkers for progression to active tuberculosis. Eur Respir J 2016; 48: 1751-1763.

18 Winje BA, White R, Syre $\mathrm{H}$, et al. Stratification by interferon- $\gamma$ release assay level predicts risk of incident TB. Thorax 2018; in press [https://doi.org/10.1136/thoraxjnl-2017-211147].

19 Andrews JR, Nemes E, Tameris M, et al. Serial QuantiFERON testing and tuberculosis disease risk among young children: an observational cohort study. Lancet Respir Med 2017; 5: 282-290.

20 Andersen P, Doherty TM, Pai M, et al. The prognosis of latent tuberculosis: can disease be predicted? Trends Mol Med 2007; 13: 175-182.

21 Ruhwald M, Aggerbeck H, Gallardo RV, et al. Safety and efficacy of the C-Tb skin test to diagnose Mycobacterium tuberculosis infection, compared with an interferon $\gamma$ release assay and the tuberculin skin test: a phase 3 , double-blind, randomised, controlled trial. Lancet Respir Med 2017; 5: 259-268.

22 Chegou NN, Heyckendorf J, Walzl G, et al. Beyond the IFN- $\gamma$ horizon: biomarkers for immunodiagnosis of infection with M. tuberculosis. Eur Respir J 2013; 43: 1472-1486.

23 Millington KA, Fortune SM, Low J, et al. Rv3615c is a highly immunodominant RD1 (Region of Difference 1)dependent secreted antigen specific for Mycobacterium tuberculosis infection. Proc Natl Acad Sci USA 2011; 108: 5730-5735.

24 Ruhwald M, de Thurah L, Kuchaka D, et al. Introducing the ESAT-6 free IGRA, a companion diagnostic for TB vaccines based on ESAT-6. Sci Rep 2017; 7: 45969.

25 Nemes E, Geldenhuys H, Rozot V. Prevention of M. tuberculosis infection with H4:IC31 vaccine or BCG revaccination. N Engl J Med 2018; 379: 138-149.

26 Van Der Meeren O, Hatherill M, Nduba V. Phase $2 b$ controlled trial of M72/AS01E vaccine to prevent tuberculosis. N Engl J Med 2018; in press [http://dx.doi.org/10.1056/NEJMoa1803484]. 